\title{
Idiopathic Retroperitoneal Fibrosis: A Retrospective Review of Clinical Presentation, Treatment, and Outcomes
}

\author{
Tanaz A. Kermani, MD; Cynthia S. Crowson, MS; Sara J. Achenbach, MS; \\ and Harvinder S. Luthra, MD
}

OBJECTIVE: To describe the clinical manifestations, laboratory results, imaging findings, and treatments in patients with idiopathic retroperitoneal fibrosis (IRF) seen at Mayo Clinic in Rochester, MN.

PATIENTS AND METHODS: In this retrospective study, we used International Classification of Diseases, Ninth Revision codes to identify all patients evaluated for IRF between January 1, 1996, and December 31, 2006, at Mayo Clinic in Rochester, MN. Medical records were reviewed, and clinical information was abstracted. Idiopathic retroperitoneal fibrosis was diagnosed on the basis of compatible imaging findings. Patients were followed up until their last visit at Mayo Clinic, death, or December 31, 2008, whichever came first.

RESULTS: Of the 185 patients identified as having IRF, 113 (61\%) were men and $72(39 \%)$ were women. Mean \pm SD age at diagnosis was $\mathbf{5 7 . 6 \pm 1 1 . 8}$ years. Biopsy specimens were obtained in $\mathbf{1 4 2}$ cases $(77 \%)$. The most common presenting symptoms were back pain $(38 \%)$ and abdominal pain $(40 \%)$. Baseline erythrocyte sedimentation rate and/or C-reactive protein levels were elevated in $88(58 \%)$ of the 151 patients tested. The median creatinine level at diagnosis was $1.3 \mathrm{mg} / \mathrm{dL}$ (interquartile range, $1.1-2.1 \mathrm{mg} / \mathrm{dL}$ ). Fifteen patients $(8 \%)$ were treated with ureteral procedures only, 58 patients $(31 \%)$ with medications only, and 105 patients $(57 \%)$ with a combination of medical and surgical therapies. Seven patients $(4 \%)$ were not treated. Corticosteroids were initiated in 116 patients (63\%), and tamoxifen was used in 120 patients $(65 \%)$. Follow-up was available for 151 patients $(82 \%)$. Creatinine levels were normal at last visit in $102(68 \%)$ of the 151 patients with follow-up. No patient developed end-stage renal disease. Relapses occurred in 18 (12\%) of the 151 patients. Eleven patients died.

CONCLUSION: In this cohort, outcomes such as end-stage renal disease or death from renal failure were not observed. Relapses may occur, and patients with IRF warrant long-term follow-up.

Mayo Clin Proc. 2011;86(4):297-303

ANA = antinuclear antibody; ANCA = antineutrophil cytoplasmic antibody; CT = computed tomography; CRP = C-reactive protein; ESR = erythrocyte sedimentation rate; IQR = interquartile range; IRF = idiopathic retroperitoneal fibrosis

$\mathrm{I}$ diopathic retroperitoneal fibrosis (IRF) includes a spectrum of diseases characterized by fibroinflammatory tissue surrounding the abdominal aorta and the iliac arteries. This process may extend into the retroperitoneum and envelop surrounding structures, often ureters. Retroperitoneal fibrosis is an uncommon condition with an estimated incidence of 1.38 cases per 100,000 people. ${ }^{1}$ Although medications, infections, malignancies, trauma, surgery, and radiation therapy have all been reported as secondary causes of this disease, most cases are idiopathic. ${ }^{2}$ Diagnosis of
IRF relies primarily on imaging studies and histopathology to rule out alternative conditions such as malignancy. Complications such as acute renal failure secondary to periureteral involvement require prompt intervention. Treatment usually involves corticosteroids with or without other immunomodulating medications or tamoxifen. In the presence of periureteral or perivascular involvement, surgical intervention may be necessary.

Many aspects of this uncommon disease are poorly understood. This retrospective study describes the clinical presentation, treatment, and outcomes in 185 consecutive patients with IRF seen at Mayo Clinic in Rochester, MN, between January 1, 1996, and December 31, 2006. To our knowledge, this is the largest single-center longitudinal study to date that includes long-term outcomes in IRF.

\section{PATIENTS AND METHODS}

Using International Classification of Diseases: Adapted Code for Hospitals codes and International Classification of Diseases, Ninth Revision codes for IRF, we identified all patients evaluated for IRF at Mayo Clinic in Rochester, MN, between January 1, 1996, and December 31, 2006. Medical records were reviewed to confirm the diagnosis. Idiopathic retroperitoneal fibrosis was defined on the basis of compatible imaging findings (retroperitoneal mass; soft tissue mass surrounding the aorta and/or adjacent tissues). Only new cases of IRF were included. Six patients with a history of IRF who had a new recurrence during the study period of interest were also included. We excluded patients who were younger than 18 years at diagnosis, prevalent cases of IRF, and patients with retroperitoneal fibrosis due to current cancer or current radiation therapy, inflammatory abdominal aortic aneurysms (without periureteral involve-

From the Division of Rheumatology (T.A.K., H.S.L.) and Division of Biomedical Statistics and Informatics (C.S.C., S.J.A.), Mayo Clinic, Rochester, MN.

Dr Kermani has received a Fellowship Award from the Vasculitis Clinica Research Consortium (National Institutes of Health National Center for Research Resources; grant number U54-RR-019497). Dr Luthra has received a generous gift from the Minnesota Arthritis Foundation.

Individual reprints of this article are not available. Address correspondence to Tanaz A. Kermani, MD, Division of Rheumatology, Mayo Clinic, 200 First St SW, Rochester, MN 55905 (kermani.tanaz@mayo.edu).

(C) 2011 Mayo Foundation for Medical Education and Research 
ment), perigraft fibrosis, or isolated sclerosing mesenteritis. The study protocol was approved by the Mayo Clinic Institutional Review Board, which waived the need for informed consent.

\section{Baseline Measurements}

Using standardized case report forms, a single rheumatologist (T.A.K.) abstracted data, including age, sex, ethnicity, date of diagnosis, date of last follow-up, vital status at last follow-up, and cause of death, if available. The presence of other autoimmune diseases or inflammatory diseases was documented. Exposure to $\beta$-blockers, asbestos, dopamine agonists, ergotamines, and methysergide was noted by a review of medication and exposure history obtained during initial evaluation for IRF. Smoking at diagnosis of IRF (never, former, current) was documented.

Baseline laboratory studies abstracted included complete blood cell count; levels of potassium, aspartate aminotransferase, alanine aminotransferase, creatinine, and Creactive protein (CRP); and erythrocyte sedimentation rate (ESR). We also gathered data on antibodies if tested at any time during evaluation.

Imaging findings at diagnosis were abstracted by review of radiology reports. Because our clinical practice is to have outside imaging studies reviewed by our radiologist, reports of outside studies were available in most cases. The type of study (computed tomography [CT] of the abdomen and pelvis, magnetic resonance imaging of the abdomen and pelvis, CT urography, or excretory urography), location of primary mass (periureteral, periaortic, both, or other), presence of hydronephrosis, and renal atrophy at baseline were collected.

Pathology reports were reviewed and the following data abstracted: method of biopsy (CT-guided or surgical), location, presence of fibrosis with inflammatory infiltrate, and immunostaining (if performed).

\section{Treatment}

All medical and surgical therapies for IRF were abstracted. Medications included glucocorticoids, tamoxifen, methotrexate, azathioprine, mycophenolate mofetil, and cyclophosphamide. Surgical interventions included placement of ureteral stents, percutaneous nephrostomy tubes, ureterolysis, and vascular stenting or surgery.

\section{Follow-up and Outcomes}

All patients in the study were followed up by medical record review until death, last visit at Mayo Clinic, or December 31, 2008, whichever came first. Only patients with 2 or more visits for IRF were included in outcome assessments. Outcome variables assessed at last follow-up and compared with baseline included clinical symptoms, mark-
TABLE 1. Demographic Information for 185 Patients With Idiopathic Retroperitoneal Fibrosis ${ }^{\text {a }}$

\begin{tabular}{|c|c|}
\hline Age at diagnosis (y) & $57.6 \pm 11.8$ \\
\hline Male sex & $113(61)$ \\
\hline \multicolumn{2}{|l|}{ Race } \\
\hline White & $138(75)$ \\
\hline African American & $10(5)$ \\
\hline Other & $7(4)$ \\
\hline Missing & $30(16)$ \\
\hline Smoking history (former or current) ${ }^{b}$ & $128(72)$ \\
\hline Time from onset of symptoms to diagnosis $(\mathrm{mo})^{\mathrm{c}}$ & $3.6 \pm 4.4$ \\
\hline Biopsy performed & $142(77)$ \\
\hline
\end{tabular}

ers of inflammation, renal function, glucocorticoid use and dose, status of other IRF treatment medications, change in size of the retroperitoneal mass as evidenced on imaging findings, and relapse. Relapse was defined as worsening of imaging findings more than 1 year after initial response to treatment.

\section{Statistical Analyses}

Descriptive statistics were used to summarize the data. Percentages were used for categorical data. Continuous variables with a normal distribution are presented as means \pm SD and those that were skewed are presented as medians with interquartile ranges (IQRs). Baseline variables of patients with vs without follow-up were compared using the student $t$ test for continuous variables and the $\chi^{2}$ test for categorical variables.

\section{RESULTS}

A medical index specialist retrieved the records of 362 patients evaluated at our institution between January 1, 1996, and December 31, 2006, for a diagnosis of IRF. We excluded 177 patients: 73 patients without IRF, 57 prevalent cases, 20 patients with a cancerous retroperitoneal mass, 12 patients with retroperitoneal fibrosis in the context of cancer or radiation therapy, 8 patients with inflammatory abdominal aortic aneurysm or perigraft fibrosis, 4 patients with isolated sclerosing mesenteritis, 1 patient with isolated sclerosing mediastinitis, and 2 patients who were younger than 18 years at diagnosis.

Our final study population included 185 patients, 113 $(61 \%)$ of whom were men and $72(39 \%)$ of whom were women; mean \pm SD age at diagnosis was $57.6 \pm 11.8$ years. Six patients $(3 \%)$ had a history of IRF with a new recurrence during our study period. Baseline demographic information is summarized in Table 1. All patients had imaging findings consistent with IRF. In 2 cases, baseline imaging was not available for review, but both cases had 
a biopsy specimen consistent with IRF. The diagnosis was confirmed by biopsy in 142 cases (77\%).

\section{Symptoms, Associated Medical Conditions, and Physical Examination Findings at Presentation}

Idiopathic retroperitoneal fibrosis was symptomatic in 166 patients (90\%) and incidentally noted on imaging in 19 patients $(10 \%)$. The most common presenting symptoms were back pain (70 cases, $38 \%$ ) and abdominal pain ( 73 cases, $40 \%$ ). Other symptoms at diagnosis are summarized in Table 2. Thirty-three patients (18\%) had exposure to a medication reported as associated with IRF, including $\beta$-blockers (29 patients, $16 \%$ ), methysergide and ergotamine (1 patient, $0.5 \%$ ), methysergide alone (1 patient, $0.5 \%$ ), methyldopa ( 1 patient, $0.5 \%$ ), and pergolide ( 1 patient, $0.5 \%$ ). Five patients (3\%) had documented asbestos exposure.

Mean \pm SD systolic blood pressure at diagnosis in 164 patients was $142 \pm 21 \mathrm{~mm} \mathrm{Hg}$, and mean \pm SD diastolic blood pressure was $80 \pm 11 \mathrm{~mm} \mathrm{Hg}$. Physical examination findings were unremarkable in most cases. Abnormal physical examination findings included lymphadenopathy (8 patients), abdominal mass ( 3 patients), abdominal bruit (6 patients), femoral artery bruit (5 patients), prominent venous collaterals (4 patients), hydrocele (3 patients), unilateral lower extremity swelling (8 patients), and bilateral lower extremity edema (24 patients).

Previous or subsequent autoimmune diseases were diagnosed in 26 patients (14\%); inflammatory bowel disease was the most common (6 patients). Eleven patients $(6 \%)$ had other fibrosing conditions, including mediastinal fibrosis in 3 patients (Table 3 ).

\section{Baseline Laboratory and Imaging Findings}

Mean \pm SD hemoglobin at diagnosis was $12.6 \pm 1.7 \mathrm{~g} / \mathrm{dL}$ (to convert to g/L, multiply by 10 ). Baseline ESR and/or CRP was elevated in 88 (58\%) of the 151 patients tested. New renal insufficiency was present in $74(42 \%)$ of the 176 patients with available creatinine measurements, and the median creatinine level was $2.3 \mathrm{mg} / \mathrm{dL}$ (IQR, 1.5-5.8 $\mathrm{mg} / \mathrm{dL}$; to convert to $\mu \mathrm{mol} / \mathrm{L}$, multiply by 88.4 ). Although autoantibodies were not systematically checked in all patients, the most commonly tested autoantibody was antinuclear antibody (ANA) in 118 patients (64\%), followed by rheumatoid factor in 89 patients (48\%). A summary of laboratory findings is provided in Table 4 .

Baseline imaging studies (within 6 months of diagnosis) were available for 169 patients (91\%). The initial imaging modality was CT of the abdomen in 148 patients (88\%), magnetic resonance imaging of the abdomen in 12 patients (7\%), and CT urography in 9 patients (5\%). The location of the retroperitoneal mass and other associated findings are provided in Table 4.
TABLE 2. Symptoms at Presentation in 185 Patients With Idiopathic Retroperitoneal Fibrosis ${ }^{a}$

\begin{tabular}{lc}
\hline Any symptoms at presentation & $166 / 185(90)$ \\
Subjective fever & $17 / 184(9)$ \\
Fatigue & $23 / 182(13)$ \\
Anorexia & $17 / 183(9)$ \\
Night sweats & $8 / 181(4)$ \\
Weight loss $>2.3 \mathrm{~kg}(5 \mathrm{lb})$ & $49 / 182(27)$ \\
Back pain & $70 / 183(38)$ \\
Flank pain & $38 / 185(21)$ \\
Testicular pain & $14 / 108(13)$ \\
Abdominal pain & $73 / 183(40)$ \\
Nausea & $37 / 181(20)$ \\
Vomiting & $24 / 181(13)$ \\
Constipation & $21 / 181(12)$ \\
New lower extremity edema & $23 / 183(13)$ \\
Lower extremity claudication & $3 / 183(2)$ \\
Arthralgias & $9 / 183(5)$ \\
\hline
\end{tabular}

${ }^{a}$ Data are presented as number (percentage) of patients. The denominator is the number of patients for which this information was available.

${ }^{\mathrm{b}}$ For testicular pain, only men were included in the denominator.

\section{Histopathologic Findings}

Pathology was available in 142 patients (77\%). Initial biopsy was CT-guided in 47 patients (33\%). Biopsy specimens showed fibrosis with inflammatory infiltrate in all patients, which is consistent with a diagnosis of IRF. Immunoperoxidase stains were performed on 37 specimens and showed polyclonal $\mathrm{CD} 3^{+} \mathrm{T}$ cells and $\mathrm{CD} 20^{+} \mathrm{B}$ cells in 30 cases.

\section{TREATMEnt}

Seven patients $(4 \%)$ received no treatment. The remaining 178 patients underwent a variety of medical and surgical interventions (Table 5). Fifteen patients (8\%) were treated with ureteral stenting or ureterolysis only, 58 patients (31\%) with medications alone, and 105 patients $(57 \%)$ with a combination of medical and surgical therapies. Corticosteroids were initiated in 116 patients (63\%) at a median dose of $60 \mathrm{mg}$ (IQR, 40-60 mg). Forty-three pa-

TABLE 3. Previous or Subsequent Associated Conditions in 185 Patients With Idiopathic Retroperitoneal Fibrosis

\begin{tabular}{ll}
\hline Inflammatory bowel disease & $6(3)$ \\
Autoimmune thyroid disease (Graves or Hashimoto disease) & $5(3)$ \\
Reidel thyroiditis & $2(1)$ \\
Inflammatory arthritis (rheumatoid arthritis or & \\
$\quad$ spondyloarthropathy) & $6(3)$ \\
Psoriasis & $2(1)$ \\
Autoimmune pancreatitis & $2(1)$ \\
Multifocal fibroinflammatory condition & $3(2)$ \\
Mediastinal fibrosis & $3(2)$ \\
Sclerosing mesenteritis & $2(1)$ \\
Giant cell arteritis or polymyalgia rheumatica & $3(2)$ \\
Other vasculitis & $2(1)$ \\
Idiopathic thrombocytopenic purpura & $2(1)$ \\
Other (idiopathic pulmonary fibrosis, sclerosing & \\
cholangitis, sarcoidosis, erythema nodosum, systemic & \\
lupus erythematosus, pseudotumor orbit) & $6(3)$ \\
\hline
\end{tabular}

Data are provided as number (percentage) unless indicated otherwise. Some patients had $>1$ associated condition. 


\begin{tabular}{|c|c|c|}
\hline & $\begin{array}{l}\text { No. } \\
\text { tested }\end{array}$ & Value \\
\hline Hemoglobin $(\mathrm{g} / \mathrm{dL})$ & 178 & $12.6 \pm 1.74$ \\
\hline Platelet count $\left(\times 10^{9} / \mathrm{L}\right)$ & 177 & $288 \pm 106$ \\
\hline Creatinine at diagnosis (mg/dL), median (IQR) & 176 & $1.3(1.1-2.1)$ \\
\hline Elevated ESR (>29 mm/hour) & 151 & $80(53)$ \\
\hline ESR at diagnosis $(\mathrm{mm} / \mathrm{h})$ & 130 & $32.3 \pm 29.1$ \\
\hline Elevated C-reactive protein (>8 mg/L) & 74 & $35(47)$ \\
\hline C-reactive protein at diagnosis $(\mathrm{mg} / \mathrm{L})$ & 70 & $20.7 \pm 26.4$ \\
\hline Renal insufficiency & 176 & $74(42)$ \\
\hline Normal TSH levels & 69 & $62(90)$ \\
\hline \multicolumn{3}{|l|}{ Autoantibody positivity } \\
\hline ANA & 118 & $6(5)$ \\
\hline Rheumatoid factor & 89 & $8(9)$ \\
\hline CCP & 29 & $0(0)$ \\
\hline p-ANCA & 37 & $6(16)$ \\
\hline c-ANCA & 37 & $0(0)$ \\
\hline MPO & 52 & $0(0)$ \\
\hline PR3 & 48 & $2(4)$ \\
\hline IgM anticardiolipin antibodies & 45 & $3(7)$ \\
\hline IgG anticardiolipin antibodies & 45 & $0(0)$ \\
\hline ENA & 75 & $0(0)$ \\
\hline Anti-dsDNA & 43 & $1(2)$ \\
\hline TPO antibodies & 13 & $5(39)$ \\
\hline \multirow{2}{*}{\multicolumn{3}{|c|}{$\begin{array}{l}\text { Baseline imaging findings } \\
\text { ( } \leq 6 \text { mo after diagnosis) } \\
\text { Location of primary retroperitoneal mass }\end{array}$}} \\
\hline & & \\
\hline Periureteral & 169 & $9(5)$ \\
\hline Periaortic and periureteral & 169 & $38(22)$ \\
\hline Other & 169 & $17(10)$ \\
\hline Abdominal aortic ectasia & 168 & $7(4)$ \\
\hline Hydronephrosis & 169 & $96(57)$ \\
\hline Bilateral hydronephrosis & 96 & $54(56)$ \\
\hline Renal atrophy & 169 & $13(8)$ \\
\hline
\end{tabular}

${ }^{a}$ Categorical data are provided as number (percentage) of patients and continuous data as mean $\pm \mathrm{SD}$, unless indicated otherwise. ANA $=$ antinuclear antibody; c-ANCA = cytoplasmic antineutrophil cytoplasmic antibody; $\mathrm{CCP}=$ cyclic citrullinated peptide; dsDNA = double-stranded DNA; ENA = extractable nuclear antigen; ESR = erythrocyte sedimentation rate; $\mathrm{IQR}=$ interquartile range; $\mathrm{MPO}=$ myeloperoxidase; p-ANCA = perinuclear antineutrophil cytoplasmic antibody; PR3 = proteinase $3 ; \mathrm{TSH}=$ thyroid-stimulating hormone; $\mathrm{TPO}=$ thyroperoxidase .

b SI conversion factors: To convert C-reactive protein values to $\mathrm{nmol} / \mathrm{L}$, multiply by 9.524 ; to convert creatinine values to $\mu \mathrm{mol} / \mathrm{L}$, multiply by 88.4 ; to convert hemoglobin values to $\mathrm{g} / \mathrm{L}$, multiply by 10 .

tients $(23 \%)$ did not receive any glucocorticoids but were placed on other medications for treatment. Tamoxifen was the most commonly used medication (120 patients, $65 \%)$, followed by methotrexate (50 patients, $27 \%$ ), azathioprine ( 9 patients, 5\%), cyclophosphamide (5 patients, 3\%), and mycophenolate mofetil (4 patients, $2 \%$ ). Other medications used were leflunomide, colchicine, imatinib mesylate, infliximab, and cyclosporin (1 case each).

\section{Outcomes}

Follow-up was available for 151 patients $(82 \%)$. When comparing the 151 patients with follow-up with the 34 patients without follow-up, no differences were observed in mean age, duration of symptom onset, proportion with biopsy, median creatinine level at diagnosis, and treatment types $(P>.05)$. A higher proportion of men than women were lost to follow-up (76\% vs 58\%; $P=.04$ ). In addition, a greater proportion of patients with vs without follow-up were white $(81 \%$ vs $41 \% ; P<.001)$.

For the outcomes section of the study, we only included the 151 patients with follow-up. Median length of followup was 4.0 years (IQR, 2.1-6.7 years). Renal function (creatinine) was normal at last visit in 102 patients $(68 \%)$. The median creatinine level at last follow-up for the group was $1.2 \mathrm{mg} / \mathrm{dL}$ (IQR, 0.9-1.3 mg/dL). No patient with followup developed end-stage renal disease. Serial imaging compared with baseline was available in 143 patients (95\%) and showed improvement in 77 patients (54\%), stable findings in 48 patients (34\%) and progression in 5 patients (3\%). In 13 cases (9\%), the retroperitoneal mass resolved (Figure 1). Fifteen patients (10\%) developed new renal atrophy.

At last follow-up, 49 (53\%) of 92 patients who had initiated corticosteroid therapy were able to discontinue the medication, whereas 43 patients (47\%) were still taking prednisone. Eighty-three (72\%) of 116 patients were still receiving treatment for IRF with other medications. Seven patients $(5 \%)$ required new stent placement after diagnosis. Ureteral stents were removed in $55(63 \%)$ of 87 patients in whom they were placed. Ureterolysis was performed after diagnosis in 27 patients (18\%). Relapses occurred in 18 patients (12\%) during the follow-up period.

After IRF diagnosis, 9 patients (6\%) developed cancers, including lymphoma $(n=3)$, prostate cancer $(n=3)$, renal cell cancer $(n=2)$, and germ cell cancer $(n=1)$. Median duration from diagnosis of IRF to cancer was 50 months (range, 4-112 months). In 2 cases, cancer was diagnosed within 1 year of IRF. One of the 2 patients was diagnosed as having

TABLE 5. Type of Medical and Surgical Therapy in 185 Patients With Idiopathic Retroperitoneal Fibrosis ${ }^{a}$

\begin{tabular}{lr}
\hline Medical intervention $^{\mathrm{b}}$ & \\
Glucocorticoids alone & $23(12)$ \\
Glucocorticoids + alternative medication & $87(47)$ \\
Tamoxifen & $120(65)$ \\
Methotrexate & $50(27)$ \\
Azathioprine & $9(5)$ \\
Cyclophosphamide & $5(3)$ \\
Mycophenolate mofetil & $4(2)$ \\
Surgical intervention & $105(57)$ \\
Ureteral stents & $77 / 105(73)$ \\
Bilateral ureteral stents & $11(6)$ \\
Nephrostomy tubes & $8 / 11(73)$ \\
Bilateral nephrostomy tubes & $52(28)$ \\
Ureterolysis & $37 / 52(71)$ \\
Bilateral ureterolysis & $12(6)$ \\
Vascular stenting or surgery & \\
\hline a Data are provided as number (percentage) of patients. & \\
${ }^{\mathrm{b}}$ Some patients received $>1$ medication.
\end{tabular}



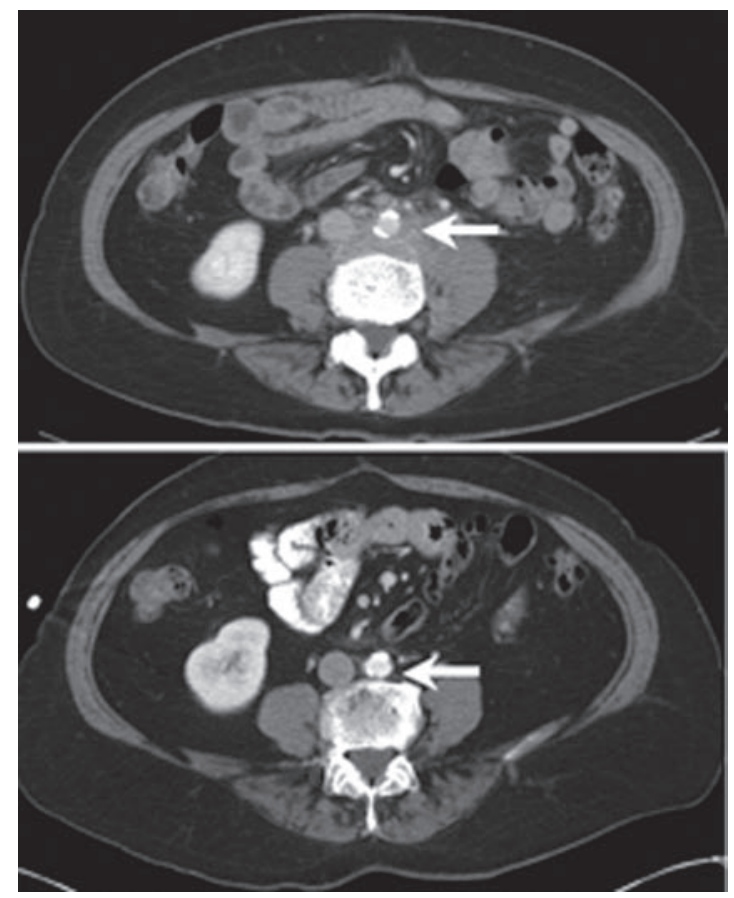

FIGURE 1. Computed tomograms demonstrating retroperitoneal fibrosis (top) with resolution after treatment (bottom).

a diffuse large B cell lymphoma 4 months after IRF, and the other developed prostate cancer 6 months after diagnosis of IRF. In the remaining 7 cases, cancer was diagnosed more than 18 months after IRF. Eleven patients died during follow-up. Causes of death were heart disease (2 patients), pulmonary disease ( 2 patients), cancer (1 patient), infection (1 patient), and unknown (5 patients). Mean \pm SD time from diagnosis to death was $3.2 \pm 3.1$ years.

\section{DISCUSSION}

Our institution is a major referral center for IRF, and one of our rheumatologists (H.S.L.) evaluated and treated more than $50 \%$ of cases seen during the study period. Patients are followed up at regular time intervals with clinical examination, laboratory testing, and serial imaging. As a result, we were able to collect information on the clinical course of the disease as well as treatments and outcomes, making this a comprehensive study that included a large number of patients. In addition to confirming previous observations on clinical features, our findings contribute to the literature by providing information on outcomes in a large series of patients with IRF.

Our inclusion criteria were based on imaging studies with characteristic findings of IRF. Histologic findings were consistent with the diagnosis in $77 \%$ of patients. We included only newly diagnosed cases, with the exception of the 6 patients (3\%) with a history of IRF who had a new recurrence during the study period. To maintain homogeneity of the study population, we excluded inflammatory abdominal aortic aneurysm, a related fibroinflammatory condition that may have a different pathogenesis from IRF. Most secondary causes of retroperitoneal fibrosis, including malignancy and retroperitoneal fibrosis in association with radiation therapy, were also excluded. We included patients with exposure to medications associated with IRF because most of them were taking $\beta$-blockers. Reports of IRF in association with $\beta$-blockers are likely confounded by the widespread use of these medications. $\beta$-Blockers may be initiated to treat hypertension before it is recognized as a feature of IRF. No conclusive evidence suggests that $\beta$-blockers are a causal agent in IRF. However, strong evidence points to methysergide as a cause of IRF, with reversal of the disease on withdrawal of the drug. ${ }^{3}$ Only 2 patients included in this study were taking methysergide. Nineteen cases with fibroinflammatory mass in other locations (Figure 2) were included because they likely represented variants of IRF. Given the atypical location of imaging findings in this subset of patients, biopsy was used to rule out an alternative process such as a malignancy in all cases.

The mean age at diagnosis in this study is similar to that in previous reports. The youngest patient in this series was aged 18 years. In contrast to other observational studies, in which a nearly 2 - to 3 -fold male predominance was reported, ${ }^{1,4-11}$ our study showed only a slight male preponderance, with a male to female ratio of 1.6:1.0. Because this is not a population-based study, drawing conclusions regarding the sex distribution in IRF is difficult. However, the previously reported male predominance may be related to sample size, inclusion of prevalent as well as incident cases, and/or differences in inclusion criteria and definition of IRF. In a recent prospective study evaluating only IRF, a roughly equal proportion of men (54\%) and women (46\%) was reported. ${ }^{12}$ Similarly, although most patients in this series were white, IRF was also observed in other racial groups, including African Americans (5\%). In the series by Scheel and Feeley, ${ }^{12} 29(60 \%)$ of the 48 patients included were white, and the remaining 19 patients were from other ethnic and racial groups.

Although markers of inflammation may be elevated in IRF, this finding is not consistent. In this series, nearly half of the patients had normal ESR and/or CRP levels at presentation. In some studies, elevated inflammatory markers have been noted in greater than $70 \%$ of patients with IRF. ${ }^{1,6,9}$ In 2 other series, lower frequencies of between $10 \%$ and $51 \%$ have been reported. ${ }^{5,13}$ On the basis of our findings, it is not unusual for patients with IRF to have normal markers of inflammation at diagnosis. There- 

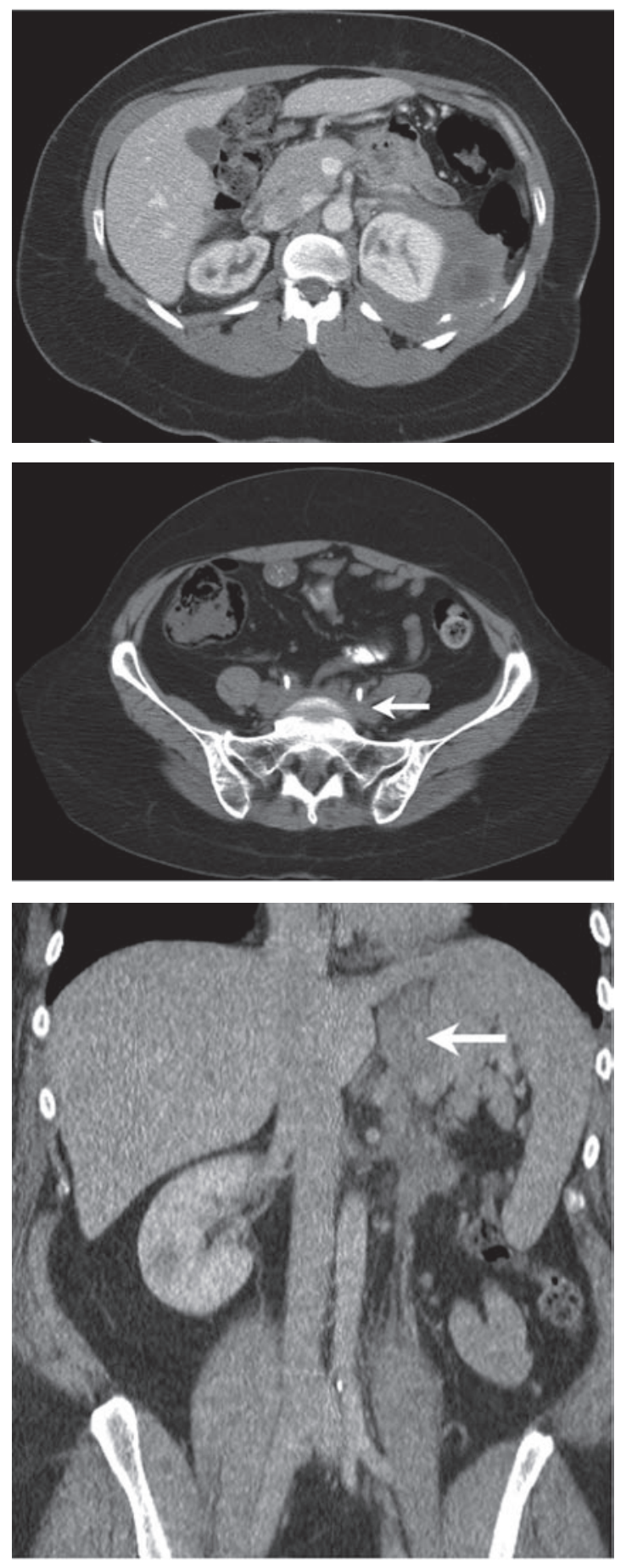

FIGURE 2. Computed tomograms showing variants of retroperitoneal fibrosis presenting as a perinephric soft tissue mass (top), a presacral mass with ureteral involvement (middle), and a left upper quadrant mass (bottom).

fore, imaging studies such as $\mathrm{CT}$ and magnetic resonance imaging remain important in diagnosing this disease. We found a lower frequency of ANA positivity as well, with only $6(5 \%)$ of 118 patients testing positive. Other investigators have reported significantly higher frequencies of ANA positivity in patients with IRF (up to $42 \%$ ). ${ }^{911}$ In 2 recent prospective studies in which ANA was systematically assessed, $25 \%{ }^{12}$ and $32 \%^{1}$ of patients tested positive for ANA. A lower frequency of ANA positivity (24\%) was also observed in another retrospective study. ${ }^{11}$ The discrepancy between our findings and those previously reported could be due to sample size or differences in patient selection, the measurement assays used, and/or the cutoff value considered positive. The next most commonly tested antibody was rheumatoid factor (9\% of patients tested positive). Given the small number of patients tested, we are unable to draw conclusions about other autoantibodies in IRF. It is interesting to note that $6(16 \%)$ of 37 patients tested positive for perinuclear antineutrophil cytoplasmic antibodies (ANCAs) without corresponding myeloperoxidase antibodies. One of the 6 patients who tested positive for perinuclear ANCAs had inflammatory bowel disease. None of the 6 patients had features of a systemic vasculitis. Biopsies of retroperitoneal tissue have yielded findings of vasculitis, ${ }^{7,9,15}$ and perinuclear and cytoplasmic ANCA positivity with and without features of systemic vasculitis have been described in association with IRF. One patient in this study had Wegener granulomatosis. Although the small number of case reports linking systemic ANCA vasculitis and IRF suggest that the 2 conditions occur together rarely, they point to a shared mechanism of pathogenesis in some cases.

Studies have suggested that IRF may be caused by a local reaction to ceroid and oxidized low-density lipoproteins found in atherosclerotic plaques. ${ }^{16,17}$ However, a systemic autoimmune process ${ }^{18}$ is suggested by a number of other findings, including systemic symptoms, association with other autoimmune diseases, presence of autoantibodies such as ANA, ${ }^{7}$ a reported association with HLADRB $1 * 03,{ }^{14}$ and the corticosteroid-responsive nature of the disease. In our study, we found associated autoimmune conditions in $15 \%$ of all patients. As in other series, autoimmune thyroid disease was one of the most commonly associated autoimmune conditions in IRF. ${ }^{1,7,12}$ In our study, inflammatory bowel disease was the most commonly associated condition.

Treatment consisted of a combination of medications and surgical intervention (ureteral stenting or ureterolysis) in half of the cases and medications alone in $31 \%$. Followup data were available for more than $80 \%$ of the patients in this study. Outcomes were evaluated retrospectively at the last visit on the basis of a combination of laboratory studies and imaging findings. The treatment of IRF was not standardized. Corticosteroids and/or tamoxifen were the most commonly used agents, followed by methotrex- 
ate, likely reflecting the bias of the treating physicians at our institution. Several agents have been used in the treatment of IRF, but the optimal treatment regimen remains to be determined. ${ }^{19}$ Although the current study design does not allow us to comment on the efficacy of specific medication regimens, most patients in this series did well, with stabilization (34\%) or improvement (approximately half) in their renal function, as evidenced by imaging studies. In 13 cases, the retroperitoneal mass resolved. Of patients in whom glucocorticoid therapy was initiated, only half were able to discontinue it. Relapses were noticed in $12 \%$ of patients during follow-up. The development of cancer after the diagnosis of IRF was unusual, and most cancers in this series were lymphomas or urologic cancers. All except 1 of these patients had biopsy specimens consistent with IRF. The median interval between IRF diagnosis and cancer development suggests that IRF was not due to cancer in most cases, although this possibility cannot be completely excluded, especially in the 2 cases in which cancer was diagnosed within 1 year of IRF. The frequency of cancer in a large cohort of patients with IRF has not been systematically assessed. Therefore, it remains unclear whether cancer risk is increased in IRF and whether it is modified by the disease itself or by its treatment.

The primary limitation of this study is its retrospective design. As a result, exposures and laboratory studies were not uniformly assessed in all patients, and information was missing in some cases. Imaging information was gathered via radiology reports rather than by actual review of the scans. However, the reports had enough detail to describe the baseline imaging findings. In the case of serial imaging, the reports contained information regarding whether findings were unchanged, worse, or improved from previous scans.

The primary strengths of the study are the large numbers of patients and the strict inclusion criteria. Other strengths include the long follow-up period (median, 4 years), which enabled us to collect follow-up information regarding treatment and outcomes in a large cohort of patients, and the objective measure (usually serial imaging) used to assess disease course and outcomes.

\section{CONCLUSION}

Idiopathic retroperitoneal fibrosis has been reported to occur more commonly in men than women, and our study confirmed this finding but found a lower male predominance than previously reported. Renal dysfunction at diagnosis was common. Autoantibodies such as ANA were infrequent in this series. However, associated autoimmune conditions were present in $15 \%$ of patients. Most patients in this study were treated with a combination of medical and surgical interventions, and the remainder were closely observed or underwent management considered appropriate by their physician. No patient progressed to end-stage renal disease. Our practice has been to follow up patients clinically and with serial imaging every 3 to 12 months to assess disease status and response to therapy. Relapses were seen in patients even after an initial response to treatment, suggesting that patients with IRF require close longterm follow-up. Further investigations are needed to determine optimal treatment, frequency of imaging to assess disease activity, duration of therapy, predictors of response to therapy, and long-term outcomes.

\section{REFERENCES}

1. van Bommel EF, Jansen I, Hendriksz TR, Aarnoudse AL. Idiopathic retroperitoneal fibrosis: prospective evaluation of incidence and clinicoradiologic presentation. Medicine (Baltimore). 2009;88(4):193-201.

2. Vaglio A, Salvarani C, Buzio C. Retroperitoneal fibrosis. Lancet. 2006;367(9506):241-251.

3. Graham JR, Suby HI, LeCompte PR, Sadowsky NL. Fibrotic disorders associated with methysergide therapy for headache. N Engl J Med. 1966; 274(7):359-368.

4. Lepor H, Walsh PC. Idiopathic retroperitoneal fibrosis. J Urol. 1979; 122(1):1-6.

5. Koep L, Zuidema GD. The clinical significance of retroperitoneal fibrosis. Surgery. 1977;81(3):250-257.

6. Baker LR, Mallinson WJ, Gregory MC, et al. Idiopathic retroperitoneal fibrosis: a retrospective analysis of 60 cases. Br J Urol. 1987;60(6):497-503.

7. Vaglio A, Corradi D, Manenti L, et al. Evidence of autoimmunity in chronic periaortitis: a prospective study. Am J Med. 2003;114(6):454-462.

8. Uibu T, Oksa P, Auvinen A, et al. Asbestos exposure as a risk factor for retroperitoneal fibrosis. Lancet. 2004;363(9419):1422-1426.

9. Corradi D, Maestri R, Palmisano A, et al. Idiopathic retroperitoneal fibrosis: clinicopathologic features and differential diagnosis. Kidney Int. 2007;72(6):742-753

10. van Bommel EF, Siemes C, Hak LE, van der Veer SJ, Hendriksz TR. Long-term renal and patient outcome in idiopathic retroperitoneal fibrosis treated with prednisone. Am J Kidney Dis. 2007;49(5):615-625.

11. Magrey MN, Husni ME, Kushner I, Calabrese LH. Do acute-phase reactants predict response to glucocorticoid therapy in retroperitoneal fibrosis? Arthritis Rheum. 2009;61(5):674-679.

12. Scheel PJ Jr, Feeley N. Retroperitoneal fibrosis: the clinical, laboratory, and radiographic presentation. Medicine (Baltimore). 2009;88(4):202-207.

13. Wagenknecht LV, Auvert J. Symptoms and diagnosis of retroperitoneal fibrosis: analysis of 31 cases. Urol Int. 1971;26(3):185-195.

14. Martorana D, Vaglio A, Greco $\mathrm{P}$, et al. Chronic periaortitis and HLA-DRB1*03: another clue to an autoimmune origin. Arthritis Rheum. 2006;55(1):126-130

15. Mitchinson MJ. The pathology of idiopathic retroperitoneal fibrosis. $J$ Clin Pathol. 1970;23(8):681-689.

16. Parums DV, Brown DL, Mitchinson MJ. Serum antibodies to oxidized low-density lipoprotein and ceroid in chronic periaortitis. Arch Pathol Lab Med. 1990;114(4):383-387.

17. Parums DV, Chadwick DR, Mitchinson MJ. The localisation of immunoglobulin in chronic periaortitis. Atherosclerosis. 1986;61(2):117-123.

18. Vaglio A, Greco P, Corradi D, et al. Autoimmune aspects of chronic periaortitis. Autoimmun Rev. 2006;5(7):458-464.

19. Swartz RD. Idiopathic retroperitoneal fibrosis: a review of the pathogenesis and approaches to treatment. Am J Kidney Dis. 2009;54(3):546-553. 